\title{
EQUALITY, DIVERSITY, AND INCLUSION
}

\author{
Marianne Egelund Siig
}

Evidence for the business case for equality, diversity, and inclusion (ED\&I) has accumulated in the last 15-20 years, erasing any doubt that having insight into these matters is salient to most aspects of your business. Whether you consider input, for example, attracting capital, talents, or customers; the efficiency in production, processes, and interactions internally; or the output/impact of your organization and its branding, products, customer relations, or effect on society, diversity, and inclusion, ED\&I has become an organisational imperative. Consequently, it is something that is now on the radar for many, if not all, stakeholders.

- Investors, suppliers, and partners have a stake in the organisation and cannot ignore the importance of your focus on these matters. Likewise, the organisation is affected by the success of the entities in which you have a stake.

- Effective risk management is also dependent on the awareness and understanding of equality and discrimination dynamics. From disruption to legislation, a diversified and inclusive organisation is essential 
for ensuring the robustness, stability, and collective intelligence to withstand and prosper.

- As one would expect, ED\&I is crucial in working with your people and culture. Being able to recognize, attract, develop, and retain diverse talent requires solid ED\&I competencies and efforts.

- ED\&I reaches way beyond human resources (HR), however. Establishing significant diversity intelligence all throughout the core business, from research and development (R\&D) to communications to product development and customer relations, will help you see more opportunities, reach more customer segments, increase customer satisfaction, and avoid pitfalls.

The importance of equality, diversity, and inclusion goes beyond the mere business case. There is an increasing demand for organisations to operate responsibly and sustainably. The expectations for transparency and engagement that today's organisations are faced with go far beyond traditional corporate social responsibility (CSR) efforts. Mere compliance is far from sufficient; organisations must be ethical in all they do, and their contributions must be meaningful, making a positive difference, but also avoiding the loss of brand value and customer loyalty when intended or unintended discriminatory practices become public.

There are increased demands for diversity-intelligent marketing and products, and the backlash companies encounter when failing stakeholders in this respect has become more far-reaching.

Heightening the diversity intelligence in a company will serve as a lever for your business, unfolding the full potential and operating in the world as a good role model showing the way for others. ED\&I strategies are not only the right thing to do but also the smart thing to do. The questions in this chapter represent by no means a comprehensive list but are meant to inspire discussions and enrich the ED\&I strategies in the company you serve as a board member.

\section{Essential questions}

- Am I, together with my governing and managerial colleagues, aware of the business case for ED\&I and the overwhelming body of scientific evidence supporting it? 
- What kind of role model is the board for equality, diversity/inclusive behaviours? (For example, is there awareness of micro-behaviours and subconscious bias, how they affect decision-making, and what should be done to counteract them?)

- Do we have gender balance (50/50 or $40 / 60$ ) in our board, C-suite, and on the VPlevel? If not, why not and what are we doing to get there?

- Do we have diversity on a broad scale (different backgrounds, ages, skin colours, nationalities, cultures, religious beliefs, sexual orientations, genders, values, personalities, physical abilities, and so on) in our board, C-suite, and on theVP level (for example, that no majority should exceed $70 \%)$ ? If not, why not and what are we doing to get there?

- Do we have an inclusive culture in which all employees can be the whole human beings they are, valued for their uniqueness and included both socially and in decision-making situations? And do our organisational structures and processes support an inclusive culture in which historical discriminatory practices are no longer reproduced?

- What would be the benefits be of seeing gender balances, equality, and D\&I as a part of our business imperative? What would it require to get there?

- Who are our "role-model" companies when it comes to ED\&I, and what would we like our company to be recognized for when it comes to ED\&I?

- How many talented individuals do the board members/the $\mathrm{CEO} /$ the executive team each know in the organisation that at least on three characteristics are different from yourselves (gender, ethnical, sexual orientation, religious belief, age, and so on)?

- If you trust in the business case for ED\&I, how much does it cost your company not to have the necessary, for example, gender balance, every year?

\section{Investors, suppliers, partners}

- How do diversity and equality considerations affect our (policy for) choice of suppliers?

- How do we ensure that our supply chain is discrimination "free"?

- What expectations regarding our diversity management efforts do our stakeholders have? 


\section{Legislation, risk, and transparency}

- How do we ensure that we are compliant with current national/ regional/supranational legislation concerning matters of equality and discrimination and that we stay up to date with new requirements?

- Have we conducted a risk assessment of factors pertaining to diversity and equality-related threats (for example, litigation costs from lawsuits, leaking of talent pipeline/inability to attract the best talent, inability to respond to [changing] customer demographics, the launching of new products that are racially/genderly, or otherwise lopsided)?

- Are we fully utilizing people analytics to create transparency concerning and guiding the ED\&I agenda (for example, splitting our people data into genders, age, and so on)? And how can we use new technologies to further advance these insights?

- How do we ensure that we offer equal pay?

- How are equality and discrimination concerns integrated into our organizational policies (for example, code of conduct, HR/people policies on harassment/employee well-being and safety, and so on)? For example, do people feel that they can safely raise their voices/opinions throughout our organisation? And do they have an easy, safe, confidential place to go if they do experience backlash/harassment/bullying/ micro-aggressions?

- What measures are we taking to eliminate gender-based (and other) discrimination already existent in our policies (for example, differences in maternity/paternity allowances, incentive- and compensation systems, and so on)?

\section{People, talent, and culture}

- When we recruit, do we look for a "good fit," or for someone who will bring something "different" or "complementary," to the table/team?

- What kind of diversity management measures have been implemented in our recruitment and talent management units (for example, analysis of where and how the pipeline is leaking, employer branding efforts to attract a diverse pool of talents, the neutralizing of gender bias in job advertisements and job descriptions, neutral job applications without 
name and photo, promotion of our company as an inclusive work-

place, affirmative/positive action recruitment policies, the minimizing of bias in screening and interview situations)?

- Are we aware of the current diversity and equality trends in society locally and abroad? (For example, are our organizational structures, processes, and culture ready to meet the demands of our future workforce, for example millennials?)

- What is our official corporate language? Our unofficial language? Do our corporate languages facilitate or impede inclusion?

- Do we offer any employee benefits supporting a gender equal workplace?

- What constitutes the "norm" in our organization, and do people who diverge from that norm feel included and valued? And do they have equal access to power and decision-making fora?

- How are we incentivizing our leaders to help us further the ED\&I agenda (for example, key performance indicators, incentives, bonus schemes, honouring, and so on)?

- How are we as board members/an organisation supporting our leaders/ specialists/HR professionals/individual contributors in fostering a gender-balanced, diverse, and inclusive workplace and environment (for example, employee resource groups, unconscious bias training)?

\section{Core business and communication}

- How are we working with the ED\&I agenda in other areas of our business than HR (for example, marketing/branding, packaging, R\&D, design, business and product development, customer service, partnerships, CSR)?

- Do we consider matters of equality, diversity, and difference in our product development and testing processes?

- How are matters of equality, diversity, and inclusion relevant to our core business areas?

- Is there any bias to be found in our branding and communication visuals and/or wordings (commercials, photos, videos, websites, catalogues/folders/flyers)? Are the heads of communications and branding/marketing sufficiently "diversity competent"? Are their staff? 


\section{Customers, community, and market growth}

- How well do our staff mirror our customer base/the society?

- How are we engaged in advancing equality in our communities (for example, sponsoring science camps for girls, lobbying for equal rights to parental leave, partnering with universities to solve the problem of the skewed pipeline, partnering with NGOs and public institutions to increase employment rates of people from minority and immigrant groups and so on)?

- How are we considering equality, diversity, and difference when we generate and use our customer/client analytics?

- Do we fully appreciate how a diversified company staff can contribute to gaining access to new customer segments (for example, via cultural insights, language competencies, networks, and so on)?

\section{Acknowledgement}

I would like to thank Sofie Skovbo Gottlieb for her help in the preparation of this chapter. 